\title{
Note sur une nouvelle station d'Anacyclus monanthos subsp. cyrtolepidioides (Pomel) Humphries dans la région du Chott el Hodna (M'sila, Algérie)
}

\section{Madani Sarri ${ }^{1}$, Djamel Sarri ${ }^{1}$, Noui Hendel ${ }^{2}$ et Hadjer Fodil ${ }^{1}$}

'Département des Sciences de la Nature et de la Vie, Faculté des Sciences, Université Mohamed Boudiaf de M'sila Département de Microbiologie et Biochimie, Faculté des Sciences, Université Mohamed Boudiaf de M’sila

\section{Correspondencia}

M. Sarri

e-mail: madani.sari@univ-msila.dz

Recibido: 27 mayo 2018

Aceptado: 18 septiembre 2018

Publicado on-line: noviembre 2018

\begin{abstract}
Note on a new station of Anacyclus monanthos subsp. cyrtolepidioides (Pomel) Humphries in the Chott el Hodna region (M'sila, Algeria)
\end{abstract}

Mots clés: Asteraceae, Anacyclus monanthos, espèce endémique, Algerie.

Key words: Asteraceae, Anacyclus monanthos, endemic species, Algeria.
Le genre Anacyclus appartient à la famille des Asteraceae, est limité à la région méditerranéenne et est particulièrement bien représentée en Algérie, le Maroc et la Tunisie (Humphries, 1979). Six espèces ont été citées par Quézel et Santa (1963), occupant plusieurs biotopes (Tableau.1).

Dans ce travail nous avons découvert une nouvelle station d'Anacyclus monanthos subsp. cyrtolepidioides (Pomel) Humphries qui a été citée en Algérie par plusieurs auteurs (Battandier et Trabut, 1902, Humphries, 1979, Quézel et Santa, 1963, Dobignard et Chatelain, 2011, The plant list, 2013). Cette espèce est endémique de l'Afrique du Nord ; en Algérie, elle occupe les pâturages arides et désertiques et sa distribution biogéographique est assez commune dans le sous-secteur des Hauts plateaux algérois et oranais (H1), le soussecteur des Hauts plateaux constantinois (H2) et le sous-secteur oriental du Sahara Septentrional (AS3) selon Quézel et Santa (1963) et selon Humphries (1979) ce taxon occupe le sud de l'Atlas Saharien (AS1, AS2, AS3 et SN2) les sols sableux.

Lors d'une sortie pédagogique destinée aux étudiants de la première année du Master en écologie des milieux naturels en avril 2017 (Université Mohamed Boudiaf de M'sila, Algérie), une nouvelle station a été observée et caractérisée par d'immenses plages d'Anacyclus monanthos

\begin{tabular}{|l|l|l|l|}
\hline \multicolumn{1}{|c|}{ Espèce } & \multicolumn{1}{|c|}{$\begin{array}{c}\text { Nom vernacu- } \\
\text { laire }\end{array}$} & \multicolumn{1}{|c|}{ Aire de répartition } & \multicolumn{1}{c|}{ Biotope } \\
\hline A. pyrethrum (L.) Cass. & Aoud el Athas & Ibéro-Maurétanien & Montagne du Tell \\
\hline A. cyrtolepidioides Pomel $(\mathbf{\square})$ & Djerf, Rebina & Endémique Nord-Africain & Pâturages arides et désertiques \\
\hline A. radiatus Lois & $\left(^{\star}\right)$ & Européen-Méditerranéen & Pâturages, clairières \\
\hline A. clavatus (Desf.) Pers. & Reliana, Bechib- & Européen-Méditerranéen & $\begin{array}{l}\text { Bords des chemins, lieux incultes, } \\
\text { champs }\end{array}$ \\
\hline A. linearilobus B. et R. & $\left(^{\star}\right)$ & Endémique & Sables littoraux \\
\hline A. valentinus L. & Ghertoufa & Méditerranéen & $\begin{array}{l}\text { Champs, lieux pierreux, pâturages } \\
\text { arides }\end{array}$ \\
\hline
\end{tabular}

$\left(^{\star}\right)$ : pas d'information ( $\left.\mathbf{a}\right)$ : ancienne nomenclature d'Anacyclus monanthos subsp. cyrtolepidioides (Pomel) Humphries selon the plant List (2013)

Tableau 1 : Caractéristiques des espèces du genre Anacyclus d’Algérie (Quézel et Santa, 1963)

Table 1: Characteristics of species of the genus Anacyclus d'Algérie (Quézel and Santa, 1963) 


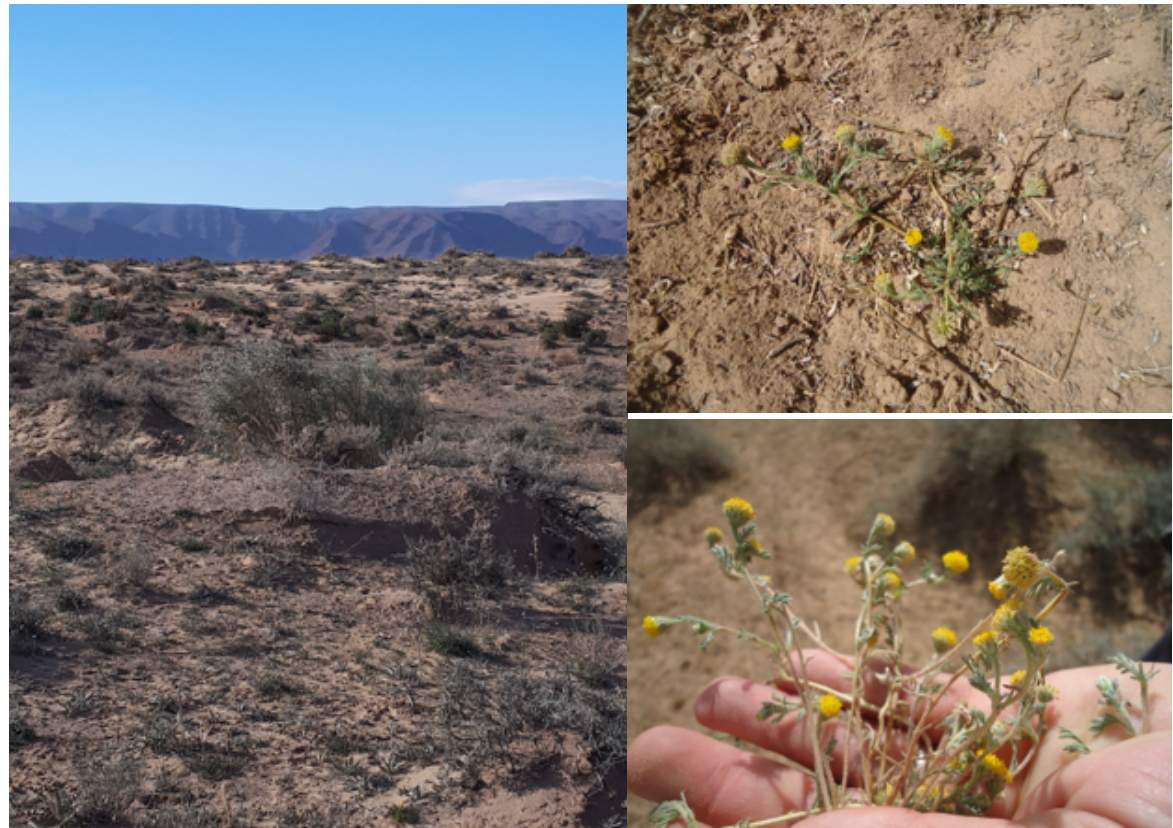

Figure 1. A gauche vue générale de la nouvelle station; à droite en haut et en bas (Anacyclus monanthos subsp. cyrtolepidioides (Pomel) Humphries)

Figure 1. Left view of the new station; right at the top and bottom (Anacyclus monanthos subsp cyrtolepidioides (Pomel) Humphries)

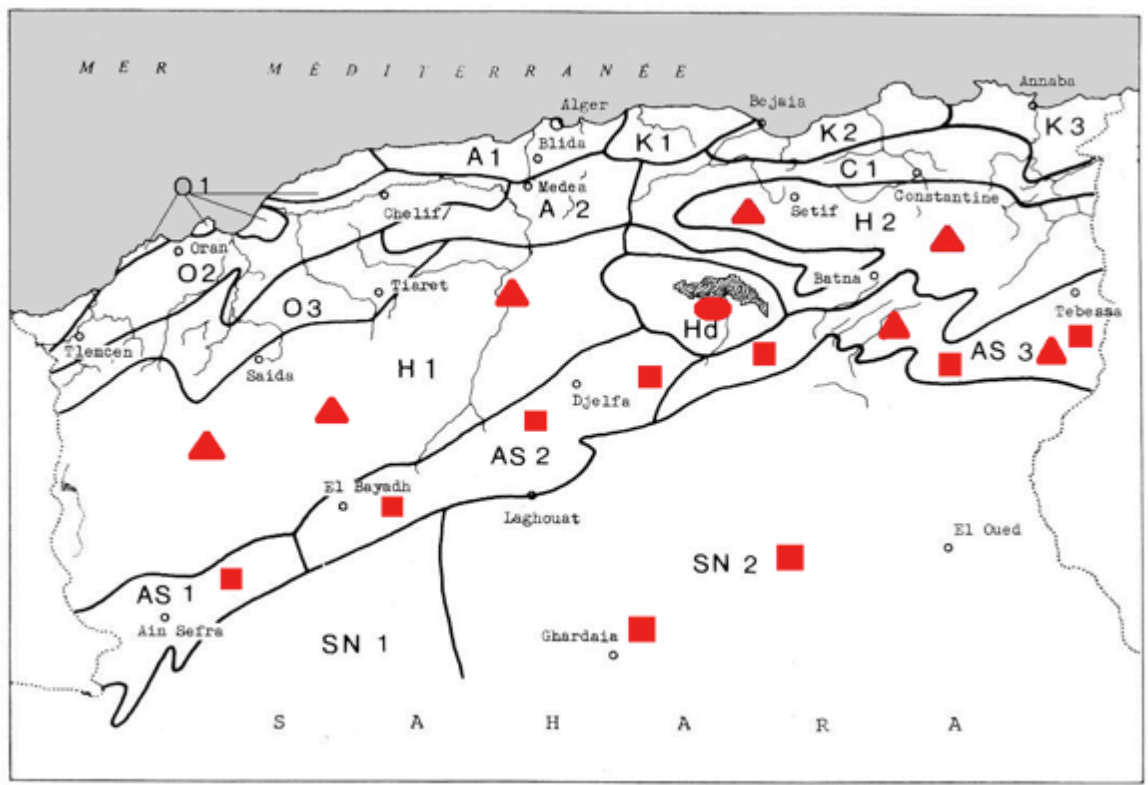

Figure 2. Carte des sous-secteurs phytogéographiques de l'Algérie du Nord (Quézel et Santa, 1962). [Cercle en rouge: notre observation en 2017 de la station nouvelle d'Anacyclus monanthos subsp. cyrtolepidioides (Pomel) Humphries dans la région du Chott el Hodna, M'sila (Algérie) ; Triangle en rouge station de Quézel et Santa (1963) et carré en rouge station d'Humphries (1979)].

Figure 2. Map of the phytogeographical subsectors of Northern Algeria (Quézel and Santa, 1962). [Circle in red: our observation in 2017 of the new station of Anacyclus monanthos subsp. cyrtolepidioides (Pomel) Humphries in the Chott el Hodna area, M'sila (Algeria); Triangle in red station Quezel and Santa (1963) and square in red station Humphries (1979)]. 
subsp. cyrtolepidioides (Figure 1) au niveau du Chott el Hodna qualifié comme zone humide par la convention RAMSAR sur la route d'EI Khobana vers Bir Hani (Figure 2). Cette station n'a pas été citée par Quézel et Santa (1963) et Humphries (1979) qui fait partie du point de vue biogéographique du sous-secteur du Hodna (Hd) de I Algerie.

\section{Références}

Battandier et Trabut (1902). Flore analytique et synoptique de l'Algérie et de la Tunisie. 460 p.

Dobignard, A. \& Chatelain, C. (2011). Index synonymique flore d'Afrique du Nord. Dicotyledoneae: AcanthaceaeAsteraceae. Editions des Conservatoire et Jardin Botaniques, Genève. Volume 2 : 145-148.

Quézel, P. \& Santa, S. (1962). Nouvelle flore de l'Algérie et des régions désertiques méridionales. CNRS, Paris, Tome 1: 11-12.

Quézel, P. \& Santa, S. (1963). Nouvelle flore de l'Algérie et des régions désertiques méridionales. CNRS, Paris, Tome 2: 978-979.

Humphries, C.J. (1979). A revision of the genus Anacyclus L. (Compositae : Anthemideae). Bull Br. Mus. Nat. Hist. (Boi.) 7 (3) : 83-142

The Plant List. (2013). Version 11. Published on the internet. [accessed January 1]. http://www.theplantlist.org/. 